\title{
Reden Sie mit!
}

\author{
Jochen Schiewe $^{1}$
}

Published online: 2 December 2021

(c) The Author(s) 2021

Wenn Sie den Deutschen Kartographie Kongress besucht haben, der im September zusammen mit der INTERGEO in Hannover stattfand, haben Sie - trotz der Masken - wahrscheinlich viele glückliche Gesichter und frohe Menschen erkennen können: Endlich wieder einmal heraus aus dem Homeoffice und endlich wieder persönliche Begegnungen mit Menschen! Wenn Sie nicht vor Ort waren, haben Sie vielleicht ähnliche Erfahrungen bei anderen Gelegenheiten machen können.

Es mag trivial klingen, dass ganz normale persönliche Begegnungen so zufrieden machen. Psychologen werden uns diesen Effekt sicherlich auch leicht erklären können. Doch die Erfahrung am eigenen Leib zeigt, dass das reale Miteinander gerade für die Entwicklung neuer Ideen und die Motivation von anderen Menschen (und sich selbst...) alternativlos sind. Begegnungen machen einfach Freude, Freude auf mehr. Im Kartographie-Umfeld freuen wir uns beispielsweise auf die International Cartographic Conference im Dezember 2021 in Florenz, den Neujahrsempfang im Januar 2022 in Berlin und den nächsten Deutschen Kartographie Kongress im September 2022 in Wien (zusammen mit den Kolleginnen und Kollegen aus Österreich, der Schweiz und - erstmals - aus Großbritannien). Detaillierte Ankündigungen und Berichte zu diesen Ereignissen werden Sie auch in den Kartographischen Nachrichten und auf der DGfK-Webseite finden.

Wir müssen uns aber auch überlegen, wie wir diese Veranstaltungen sowie die der Sektionen und Kommissionen künftig gestalten. Ich hatte bereits im Editorial des Heftes 2/2021 darauf hingewiesen, dass wir jeweils Alleinstellungsmerkmale für digitale, hybride und Präsenz-Angebote schaffen müssen. Gerade für PräsenzAngebote müssen immer mehr Arbeitgeber überzeugt werden, dass eine Dienstreise zwingend notwendig ist

Jochen Schiewe

jochen.schiewe@hcu-hamburg.de

1 HafenCity Universität Hamburg, Hamburg, Germany bzw. eine Online-Teilnahme allein nicht ausreichend ist. Wir müssen also über Themen, Formate, Orte und Beitragende nachdenken. Mit „wir “ meine ich ausdrücklich auch Sie! Teilen Sie mir doch bitte Ihre Ideen und Wünsche mit.

Die Erfahrungen aus den letzten Monaten haben auch gezeigt, dass wir nicht nur Nachholbedarf in der persönlichen Kommunikation haben. So hat sich der DGfKVorstand vorgenommen, die Öffentlichkeitsarbeit über alle vorhandenen Kanäle (Newsletter, E-Mail, Webseite, Twitter, Facebook, usw.) zukünftig zu verstärken. Aber auch hier gilt: Wir können einen Rahmen aufmachen - einen echten Austausch gibt es nur, wenn „wir “ alle mitmachen!

Dabei gibt es viele Themen zum Austauschen: Neben der Gestaltung unserer Veranstaltungen gehören Inhalte und Aufmachung der KN (speziell des Informations- und Praxisteils), die Gewinnung junger (und älterer!) Mitglieder sowie die Motivation zur ehrenamtlichen Mitarbeit in Sektionen und Kommissionen dazu. Wir werden uns im Januar 2022 auch wieder zu einem Klausurtag in größerer Runde treffen, bei dem nicht nur Probleme benannt, sondern vielmehr Lösungen angeschoben werden sollen. Nochmals: Teilen Sie mir doch bitte Ihre Ideen und Wünsche mit. Reden Sie mit!

\section{Unsere Verbandsgeschichte}

Apropos: Mein Beitrag zur Vorgängergesellschaft der DGfK, der Deutschen Kartographische Gesellschaft (D.Kart.G.) zwischen 1937 und 1949 (KN 4/2020), ist auf eine erfreuliche Resonanz gestoßen. Dieser Artikel sollte und konnte aber nur ein Auslöser sein. Um eine weitere und systematische historische Aufarbeitung und Bewertung unserer Verbandsgeschichte zu erhalten, startet der Vorstand der DGfK in dieser KN-Ausgabe einen Aufruf, um Personen mit Interesse und Wissen für diese Aufgabe zusammenzubringen. Und noch einmal: Machen und reden Sie mit! 


\section{Alles Gute für 2022!}

Liebe Mitglieder der DGfK, liebe Kartographie-Begeisterte! Am Ende dieses Jahres möchte ich mich auch im Namen des gesamten Vorstandes für Ihre Unterstützung bedanken - inklusive der immensen Zustimmung, die wir bei den Vorstandswahlen durch Sie erfahren haben. Mein persönlicher Dank geht insbesondere an meine Kollegin und meine Kollegen im Vorstand für die überaus angenehme und vertrauensvolle Zusammenarbeit.

Im Namen des gesamten DGfK-Vorstandes wünsche ich Ihnen allen und Ihren Familien frohe Festtage und ein gesundes sowie kommunikatives Jahr 2022!

\section{Jochen Schiewe.}

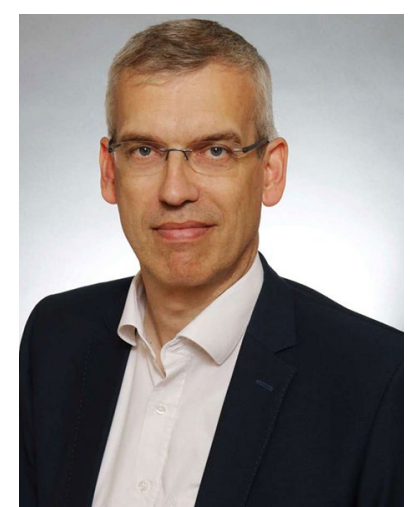

Supplementary Information The online version contains supplementary material available at https://doi.org/10.1007/s42489-021-00093-0.

Funding Open Access funding enabled and organized by Projekt DEAL.

Open Access This article is licensed under a Creative Commons Attribution 4.0 International License, which permits use, sharing, adaptation, distribution and reproduction in any medium or format, as long as you give appropriate credit to the original author(s) and the source, provide a link to the Creative Commons licence, and indicate if changes were made. The images or other third party material in this article are included in the article's Creative Commons licence, unless indicated otherwise in a credit line to the material. If material is not included in the article's Creative Commons licence and your intended use is not permitted by statutory regulation or exceeds the permitted use, you will need to obtain permission directly from the copyright holder. To view a copy of this licence, visit http://creativecommons.org/licenses/by/4.0/. 